\title{
Heavy isotopes cosmic ray spectrometer (HICRS) for the NUCLEON-2 mission
}

\section{Karmanov}

SINP MSU

Skobeltsyn Institute of Nuclear Physics, Moscow State University, Moscow, Russia

E-mail: karmanov68@mail.ru

\section{Kovalev}

SINP MSU

Skobeltsyn Institute of Nuclear Physics, Moscow State University, Moscow, Russia E-mail: im.kovalevaphysics.msu.ru

\section{A. Kurganov ${ }^{1}$}

SINP MSU

Skobeltsyn Institute of Nuclear Physics, Moscow State University, Moscow, Russia E-mail: me@sx107.ru

\section{Panasyuk}

SINP MSU

Skobeltsyn Institute of Nuclear Physics, Moscow State University, Moscow, Russia E-mail: panasyukesinp.msu.ru

\section{A. Panov}

SINP MSU

Skobeltsyn Institute of Nuclear Physics, Moscow State University, Moscow, Russia E-mail: panovenator@gmail.com

\section{Podorozhny}

SINP MSU

Skobeltsyn Institute of Nuclear Physics, Moscow State University, Moscow, Russia E-mail: dmpeeas.sinp.msu.ru

\section{G. Sedov}

SINP MSU

Skobeltsyn Institute of Nuclear Physics, Moscow State University, Moscow, Russia E-mail: sge4440ya.ru

\section{Tkatchev}

SINP MSU

Skobeltsyn Institute of Nuclear Physics, Moscow State University, Moscow, Russia E-mail: ptkatcheveyandex.ru

\footnotetext{
${ }^{1}$ Speaker
} 


\section{A. Turundaevskiy} SINP MSU

Skobeltsyn Institute of Nuclear Physics, Moscow State University, Moscow, Russia E-mail: anteeas.sinp.msu.ru

The NUCLEON-2 experiment is aimed at the investigation of isotope and charge composition of medium, heavy and ultra-heavy ions $(\mathrm{Z}<82)$ in the $300 \mathrm{MeV} / \mathrm{N}-1 \mathrm{GeV} / \mathrm{N}$ energy range. The concept design of HICRS for the NUCLEON-2 satellite cosmic ray experiment is presented. The performed simulation confirms the isotope resolution algorithms and techniques.

35th International Cosmic Ray Conference - ICRC2017

10-20 July, 2017

Bexco, Busan, Korea 


\section{Introduction}

\subsection{Scientific problems connected with the isotope composition of GCR}

In the current state of space-related fields of physics, including physics of the origin of cosmic rays and astrophysics in general, data on the isotope composition is of considerable interest. This data affects a wide variety of physical problems.

The fluxes of superheavy nuclei in galactic cosmic rays (GCR) are sensitive to the local environment of the Sun, as their free paths are relatively short ( 1kpc@ $@>40)$ before they fragment. Therefore, by measuring the abundances of secondary isotopes one can determine the local diffusion coefficient, knowledge of which is crucial, particularly, for the correct description of positron and electron fraction in cosmic rays. Using many convenient radioisotope clocks among isotopes of superheavy nuclei their age can also be determined. All there measurements are of great importance for the investigation of such exotic and nonstandard sources as neutron stars and dark matter.

Supernova explosions in a number of current models may occur in heavy elementsenriched medium formed by earlier supernova explosions and stellar winds, rather than in a standard interstellar medium. This process may lead to a variety of anomalies in isotope and charge composition of GCR that require detailed examination. Models of non-standard cosmic ray acceleration mechanisms in OB stellar associations where the abundance of heavy nuclei in cosmic rays serves as a marker of these mechanisms must also be studied.

From the viewpoint of nucleosynthesis mechanisms examination the data on the isotope composition of cosmic rays is also very important in charge range from $Z=40$ to 65 , as this range contains the double peak of element abundance corresponding to the fast r-process of neutron capture and the slow s-process.

\subsection{Existing data}

A vast majority of data on fluxes of heavy and superheavy nuclei was obtained in four experiments: LDEF, HEAO-C3, SuperTiger and ACE/CRIS.

The LDEF experiment [1] was based on long-term exposure of a solid-state track detector in open space. The experiment was designed to determine the chemical composition of lowenergy GCRs in the subactinide $(70 \leq Z \leq 87)$ and actinide $(88 \leq Z \leq 103)$ regions. It recorded 35 actinide events in the energy range of $1-2 \mathrm{GeV} /$ nucleon and acquired a great many statistics in the subactinide region. The abundances of superheavy elements in GCRs and the Solar System were compared.

The $\mathrm{C} 3$ detector at the HEAO-3 astronomical observatory [2] was based on a combination of Cherenkov counters, gas proportional counters, and hodoscopes from multi-wire ionization chambers. The charge spectrum of superheavy nuclei in the charge range of 40 to 62 was determined. A plateau in the abundance of different nuclei was detected in the charge range of 44 to 60 . In this region, the experiment recorded from 10 to 30 events for the main even nuclei.

The balloon-borne superTIGER stratospheric experiment [3] was also based on using the Cherenkov technique. Charge distributions in a wide range of charges for superheavy nuclei with charges above $Z=40$ were obtained for nucleus energies above 2-3 GeV/nucleon, with 
statistics comparable to those obtained earlier in the HEAO-3-C2 experiment. None of the experiments [1-3] measured the isotopic composition of cosmic-ray nuclei.

Information on isotopes of superheavy nuclei in cosmic rays was obtained in the CRIS experiment on board the ACE spacecraft [4]. The experiment used an approach based on recording the Bragg peak of the complete stopping of nuclei in an array of thin silicon detectors. The spectrometer was carried into orbit in 1997 and is still in operation. The CRIS spectrometer obtained unique charge spectra and isotope compositions of super-heavy nuclei up to $Z=32$ for energies of several hundreds of $\mathrm{MeV} /$ nucleon. It should be emphasized that there are no experimental data on the isotopic composition of nuclei with $Z>32$, therefore obtaining of such data is extremely important.

\subsection{Proposed mission}

Since no experiments are planned for the investigation of the described scientific problems in the near future and a lack of experimental data on the isotope and charge compositions of cosmic rays at $Z>32$ for isotope composition and $Z>40$ for charge composition is present, a new experiment is needed for the continuation of studies in these fields. Considering the decreasement of GCR flux intensity with increasing charge, an experiment with exposure several magnitudes larger than of the ACE/CRIS one's is needed.

\section{The NUCLEON-2 Mission}

\subsection{Description}

The NUCLEON-2 mission is a proposed satellite experiment project for direct measurement of cosmic rays for the investigation of isotope and charge composition. The $\mathrm{Z}$ range for charge composition measurement is 7-94; for the isotope composition it is 7-66. The exposition time is not less than 5 years with a planned geometric factor of $1.5 \mathrm{~m}^{2} \mathrm{sr}$.

The separation in the experiment uses a modified E-dE telescope technique. This method is based on measuring the particle's total energy deposit $\mathrm{E}$ and the energy loss $\mathrm{dE} / \mathrm{dx}$ in one of the spectrometer's detector. The product of these two variables $\mathrm{E}(\mathrm{dE} / \mathrm{dx})$ is proportional to the particle mass $\mathrm{M}$ and square of the particle charge $\mathrm{Z}$.

The energy range of the spectrometer is $0.1-1 \mathrm{GeV}$, and the energy measurement error does not exceed $0.4 \%$. The requirements for precision are determined by the Rayleigh criterion in separating

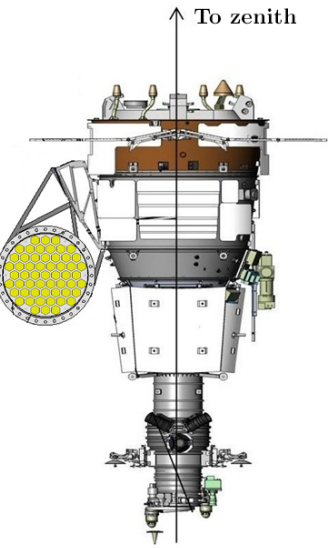

Fig. 1: Possible arrangement of the NUCLEON-2

Experiment neighboring isotopes up to nuclei with $\mathrm{Z}=60$.

To minimize the expenses it was proposed that the NUCLEON-2 scientific equipment will be launched as a payload on a russian commercial satellite. Mathematical simulations show that at orbits above $400 \mathrm{~km}$ the most optimal orientation for a two-sided detector is to orient it horizontally with respect to the Earth. The possible arrangement of the NUCLEON-2 experiment is shown at fig. 1 . 


\subsection{Construction of the experiment and HICRS}

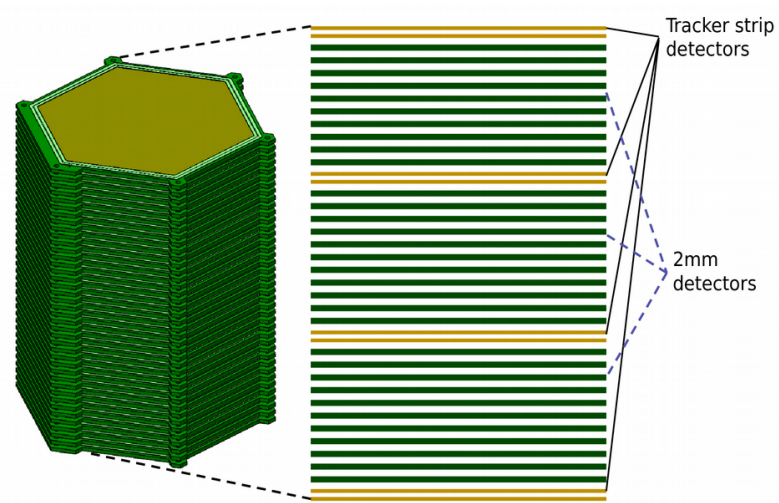

Fig. 2: HICRS construction

Scientific equipment of the experiment consists of 55 identical Heavy Isotope Cosmic Ray Specrtometers (HICRS). The principles of operation, control, and monitoring would be taken from the NUCLEON scientific equipment as much as possible [5]. The basic characteristics of the NUCLEON-2 scientific equipment are a mass of no more than $330 \mathrm{~kg}$, the device is placed in a cylindrical vessel with dimensions no more than $\mathrm{D} \sim 1500 \mathrm{H} \sim 360$ $\mathrm{mm}$, no more than 27 thousand amplitude channels, and energy consumption no higher than 320 W.

Each HICRS is a hexagonical stack of silicon detectors - 32 2mm-thick main detectors and 8300 um-thick tracker strip detectors (fig. 2) with two symmetric entrance windows at the top and bottom. By measuring the particle's energy deposit in each detector untill it stops with a corresponding bragg peak in the spectrometer the particle charge and mass are determined using E-dE technique, which is applied multiple times, therefore increasing the accuracy.

\subsection{Expected results}

\begin{tabular}{|c|c|c|c|}
\hline Nucleus, $Z$ & N & Nucleus, $Z$ & N \\
\hline Fe 26 & $3 \cdot 10^{7}$ & Ho, 67 & 24 \\
\hline Co 27 & $1.4 \cdot 10^{\circ}$ & Er, 68 & 100 \\
\hline Ni 28 & $1.1 \cdot 10^{\circ}$ & Tm, 69 & 29 \\
\hline Cu 29 & $1.6 \cdot 10^{4}$ & Yb, 70 & 110 \\
\hline Zn 30 & $1.6 \cdot 10^{4}$ & Lu, 71 & 45 \\
\hline Ga 31 & 2000 & Hf, 72 & 90 \\
\hline Ge 32 & 2300 & Ta, 73 & 53 \\
\hline As 33 & 350 & W, 74 & 100 \\
\hline Se 34 & 1400 & Re, 75 & 78 \\
\hline Br 35 & 200 & Os, 76 & 140 \\
\hline Kr 36 & 830 & Ir, 77 & 145 \\
\hline Rb 37 & 250 & Pt, 78 & 220 \\
\hline Sr 38 & 1000 & Au, 79 & 160 \\
\hline Y 39 & 250 & Hg, 80 & 150 \\
\hline Zr 40 & 500 & Tl, 81 & 85 \\
\hline Nb 41 & 150 & Pb, 82 & 155 \\
\hline Mo 42 & 230 & Bi, 83 & 62 \\
\hline Ru44 & 100 & Po, 84 & 75 \\
\hline Ag 47 & 140 & At, 85 & 76 \\
\hline Cd48 & 120 & Rn,86 & 30 \\
\hline Sn 50 & 120 & Fr, 87 & 3 \\
\hline Te 52 & 140 & Ra, 88 & 12 \\
\hline Xe 54 & 80 & Ac, 89 & 3 \\
\hline Ba 56 & 180 & Th, 90 & 13 \\
\hline Ce 58 & 50 & Pa, 91 & 2 \\
\hline Nd60 & 40 & U,92 & 53 \\
\hline Dy 66 & 180 & ??? & $? ? ?$ \\
\hline & & & \\
\hline
\end{tabular}

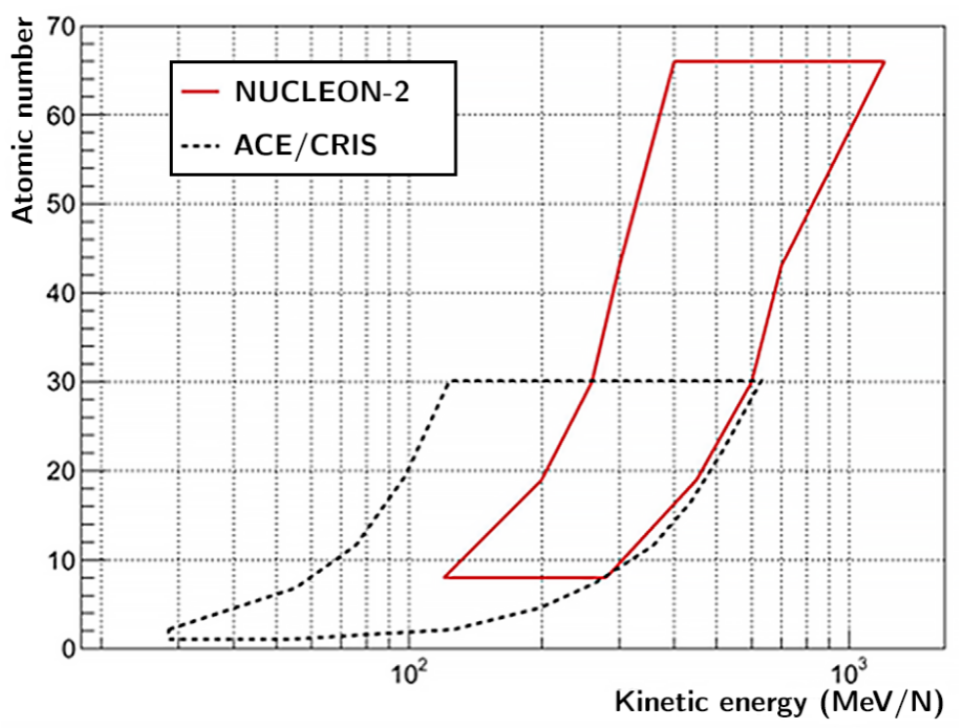

Fig. 3: Expected mission statistics (on the left, green for isotopic and charge resolution, yellow only charge) and energy range (on the right)

The expected experiment statistics are shown on the fig. 3. On it's left is a table showing expected statistics from 5 years of exposition. The planned geometric factor with this exposition time yields an exposure 20 times larger than of the CRIS ACE experiment. 
On the right side of the fig. 3 the NUCLEON-2 mission energy range (depending on the particle atomic number Z) is given in comparsion with the CRIS ACE experiment one's.

\section{The prototype and simulation results}

\subsection{The prototype and CERN test}

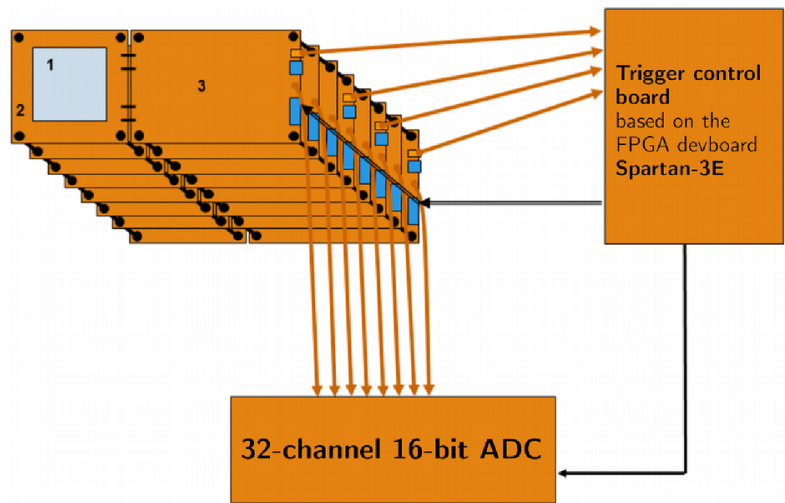

Fig. 4: Prototype block diagram

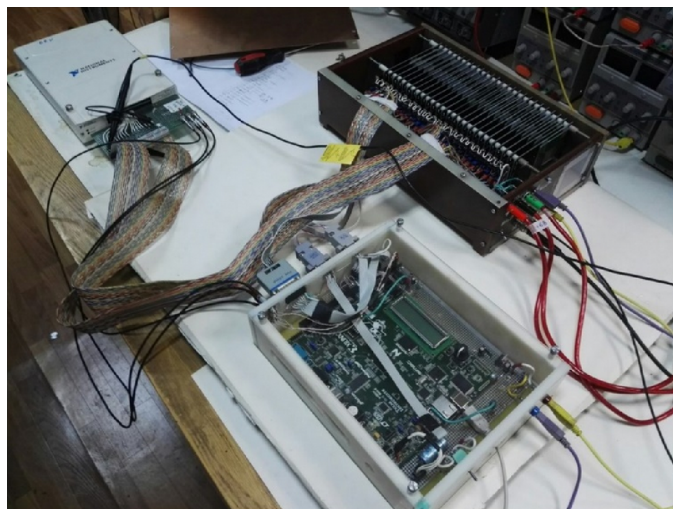

Fig. 5: Prototype photo

A prototype of the HICRS spectrometer was developed. The prototype consists of 13 $1 \mathrm{~mm}$-thick and 13 300um-thich detectors, giving 26 detectors in total. Each detector is connected throw a preamplifier board to a 32-channel 16-bit ADC, while some of the 300umthick detectors are connected throw a similar front-end preamplifier to a FPGA trigger controller board based on the Spartan-3E devboard (fig. 4). A photo of the prototype is shown on fig. 5.

The prorototype was tested on the SPS accelerator in CERN with a beam of nucleus at 150 $\mathrm{GeV} / \mathrm{N}$ and $\mathrm{Z}$ up to 82 . The results of this test confirm the proposed experiment's $\mathrm{Z}$ range from $Z=3$ up to $Z=82$ and are shown on the fig. 6 .
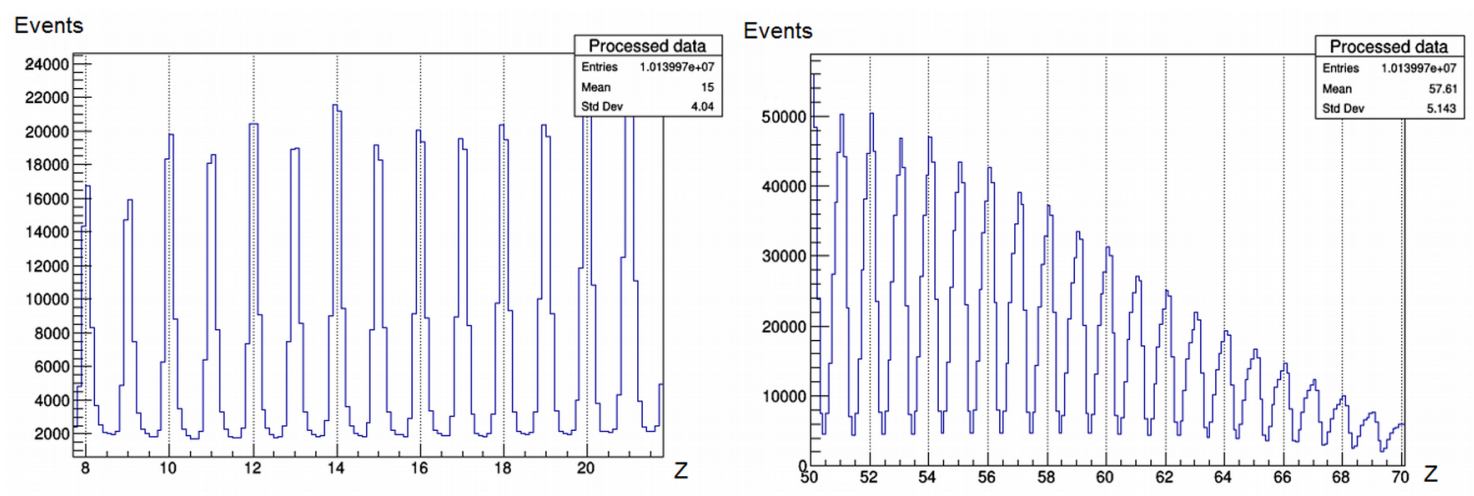

Fig. 6: CERN test results

\subsection{Monte-carlo simulation}

A monte-carlo simulation was performed in GEANT3, GEANT4 and FLUKA codes separately to test the experiment's isotope resolution, optimise the experiment's dimensions and to obtain the acceptable electronics noise level. The model construction consists of $900100 \mathrm{um}-$ 


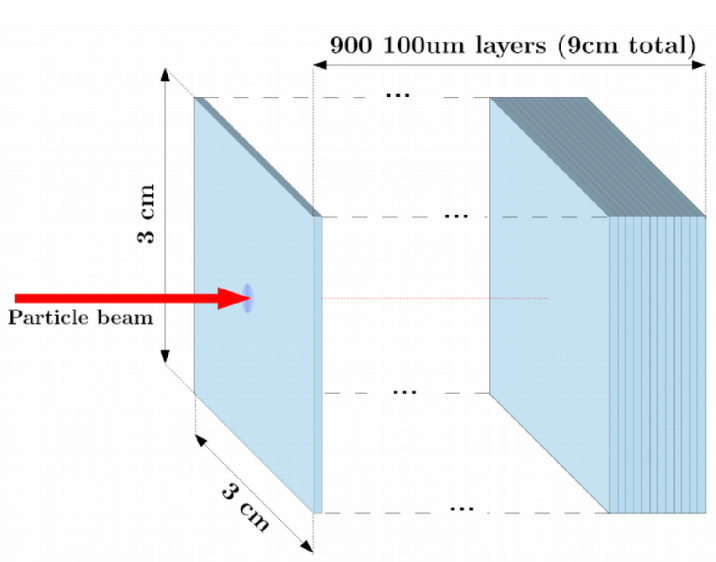

Fig. 7: Model construction thick detectors (fig. 7), which are laterly combined into layers of greater thickness after the simulation. This combination of layers is required to give the possibility of optimising the experiment's construction without the need of resimulation. Yet relatively simple, this model still shows basic relations and therefore can be used for the soluton of the described simulation tasks.

Simulation data was then analysed using different processing methods (neural networks and multidimensional analysis based on the maximum likelihood method). Only a weak model dependence was demonstrated by models comparison, and a reliable isotope separation up to $\mathrm{Z}=64$ was obtained.

The separation results for molybdenium and tin isotopes are shown on the fig. 8 .
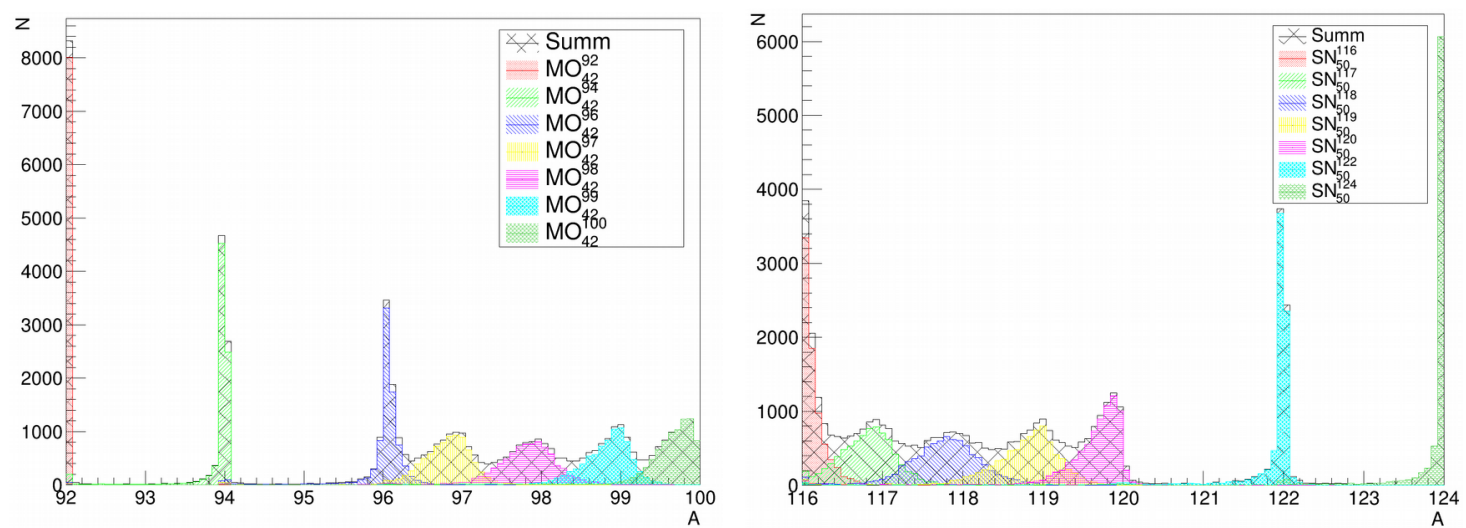

Fig. 8: Monte-carlo simulation analysis results for molybdenium and tin isotopes at zero noise

\section{Conclusion}

The NUCLEON-2 experiment is a planned experiment for the investigation of isotope composition of superheavy nuclei in GCR. The NUCLEON-2 experiment concept design is presented, as well as the prototype beamtest and simulation results, both of which confirm the validity and operability of the main ideas laid in the detector. The experiment is still in development and will be launched in the year 2020-2022.

\section{Acknowledgments}

We are grateful to ROSCOSMOS State Space Corporation and Russian Academy of Sciences for their continued support of this research.

\section{References}

[1] Donnelly, J., Thompson, A., O’Sullivan, D., et al., Astrophys. J., 2012, vol. 747, p. 40. 
[2] Binns, W.R. Garrard, T.L., et al., Astrophys. J., 1989, vol. 346, p. 997.

[3] Binns, W.R., Bose, R.G., Braun, D.L., et al., Astrophys. J. Lett., 2014, vol. 788, p. 18.

[4] Israel, M.H., Binns, W.R., Christian, E.R., et al., Proc. 32nd Int. Cosmic Ray Conf., Beijing, 2011, vol. 6, p. 32 .

[5] Atkin, E., Bulatov, V., Dorokhov, V., et al., Nucl. Instrum. Methods Phys. Res., Sect. A, 2015, vol. 770 , p. 189.

[6] Measuring the Isotopic Composition of Superheavy Nuclei of Galactic Cosmic Rays in the NUCLEON-2 Experiment, Kurganov A.A., Panasyuk M.I., Panov A.D., Podorozhny D.M., Turundaevskiy A.N. IZVESTIYA ROSSIISKOI AKADEMII NAUK, SERIYA FIZICHESKAYA, vol. 81 , № 4, p. 436-438 